\title{
VOLTAGE CONTROL DEMONSTRATION FOR LV NETWORKS WITH CONTROLLABLE DER - THE SUSTAINABLE PROJECT APPROACH
}

\author{
Hélder COSTA \\ INESC TEC - Portugal \\ helder.m.costa@inesctec.pt \\ Luís SECA \\ INESC TEC - Portugal \\ luis.seca@inesctec.pt \\ Ricardo SANTANA \\ EDP Distribuição - Portugal \\ Ricardo.Santana@edp.pt \\ Luís ROSA \\ EDP Distribuição - Portugal \\ LuisMiguel.Rosa@edp.pt
}

\author{
Miguel MIRANDA \\ INESC TEC - Portugal \\ luis.m.miranda@inescporto.pt \\ André MADUREIRA \\ INESC TEC - Portugal \\ andre.g.madureira@inesctec.pt \\ Miguel LOURO \\ EDP Distribuição - Portugal \\ Miguel.Louro@edp.pt \\ Nuno SILVA \\ EFACEC - Portugal \\ nuno.silva@efacec.com
}

\author{
João RAMOS \\ INESC TEC - Portugal \\ jcramos@inesctec.pt \\ Diogo LEMOS \\ EDP Distribuição - Portugal \\ Diogo.Lemos@edp.pt \\ Pedro G. MATOS \\ EDP Distribuição - Portugal \\ Pedro.GodinhoMatos@edp.pt
}

\begin{abstract}
One of the main constraints for Renewable Energy Sources (RES) integration in LV networks are overvoltages caused by changing the normal power flow of the network. In favourable weather conditions high voltages may lead to overvoltage trips thus preventing the injection of renewable energy into the grid.

An optimized management of power injection from controlled RES to keep the grid voltage within regulatory limits enables a larger energy output and deployment of distributed generation. The SuSTAINABLE project developed a centralized algorithm based on a hierarchical methodology to control distributed power injection and solve the identified issue. A decentralized algorithm based on a coordinated droop control embedded in the inverters was developed as well. In order to evaluate the proposed algorithms a controllable $P V \mu G$ and batteries were installed at the end of the feeder of a real LV network operated by EDP Distribuição. The obtained results are presented in this paper and show that a hierarchical methodology to control power injection could optimize RES energy production while maintaining voltages within bounds, thus enabling a larger deployment of RES at the LV levels.
\end{abstract}

\section{INTRODUCTION}

The deployment of Distributed Energy Resources (DER) in electric distribution systems in a safe and efficient way has been fostering the development of the smart grid concept and associated controls for managing grid operation.

The SuSTAINABLE project has been developing and demonstrating a new operation paradigm, leveraging information from smart meters and short-term localized predictions to manage electrical distribution systems in a more efficient way, enabling a large-scale deployment of variable, renewable-based Distributed Generation (DG). The advanced control architecture for the SuSTAINABLE project aims at enabling a coordinated and efficient control of the whole electrical distribution system, taking advantage of its own resources in order to support network operation [1].

One of the issues that limits the installation of Renewable Energy Sources (RES) in distribution networks, especially at the Low Voltage (LV) level, is the voltage rise effect that occurs in situations where there is significant power injection from these dispersed sources.

In the SuSTAINABLE project it is proposed to tackle this issue by developing a hierarchical methodology to control the power injection from RES thus maintaining the grid voltage within regulatory limits. In [1], a general overview of this approach is included while in [2] the voltage control tool developed for Medium Voltage (MV) networks is presented that exploits distributed storage devices, DG units and transformers with On-Load Tap Changing (OLTC) capability.

However, at the LV level there are several specific limitations. Firstly, LV networks are intrinsically unbalanced since many loads and generation units are single-phase, which requires a three-phase analysis. Another significant main obstacle to an efficient operation of the LV distribution system has to do with the fact that these grids are often poorly characterized both in terms of topology and electrical characteristics of the lines. In some cases, the only information available is the knowledge of which loads connect to which MV/LV distribution transformer.

In order to ensure a higher level of flexibility, envisaging the evolution of smart grids, controllable small scale storage systems are also considered. Both decentralized and centralized control strategies are being tested as part of the project. Two centralized algorithms for voltage control in LV grids were developed for this purpose and integrated into LV automation systems.

The proposed voltage control approach was tested using a facility with several controllable DER facility built in a real LV network operated by EDP Distribuição. Both centralized and decentralized algorithms for voltage control were tested for a period of several months. The decentralized algorithm used consists on a coordinated droop control embedded in the inverters of the different DER in order to adjust the active power injected when 
the voltage goes beyond a configurable threshold. The proposed centralized algorithm, housed in the Distribution Transformer Controller (DTC), is based on set of rules that use a merit order of the DER that are available for control actions based on real-time system state and measurements, as well as distance factors. To obtain the real-time measurements dedicated smart meters had to be installed at the customers' premises.

Results from field test are presented in this paper that demonstrate how a hierarchical distributed architecture can leverage the role of controllable DER in facilitating the increase of RES at the LV.

\section{CENTRALIZED VOLTAGE CONTROL ALGORITHM}

The proposed methodology aims at providing a real time solution in order to control voltage deviations in $\mathrm{LV}$ grids based on a set of rules that is able to manage all the controllable grid assets according to a merit order.

The selected control actions are prioritized according to the type of controllable resources that are available in the grid according to the following priority list for grid resource's actuation:

1. Energy storage devices;

2. OLTC transformers at the $\mathrm{MV} / \mathrm{LV}$ secondary substation;

3. Micro-generation units;

4. Controllable loads under demand response schemes.

For each type of controllable grid asset, the control action is determined taking into account a set of decision factors that prioritize which is the unit best suited to solve a specific voltage violation. Some of the most relevant decision factors are the proximity to voltage violation location, the flexibility of operation and the potential impact in mitigating the voltage deviation.

The proposed methodology (shown in Figure 1) is adaptive since it is capable of solving voltage problems taking advantage of the available distributed resources in two distinct situations [3]:

- Full knowledge of the LV grid: Topology and access to smart metering devices and possibility of running a three-phase unbalanced power flow routine.

- Limited knowledge of the LV grid: Unknown topology; access only to smart meter readings and geographic coordinates of customers.

The voltage control algorithm is scheduled to run periodically following each update of the grid's condition (i.e. every 5 or 15 minutes, depending on the cycle of measurement of the main quantities such as power injections at each node).

Whenever a violation is detected, if there is sufficient grid information for running a "smart" three-phase power flow, a suitable solution for controlling voltage profiles is determined by testing several possible solutions iteratively and then identifying which resources need to be actuated in order to solve the voltage violation.

With limited information and without the possibility of running a power flow, the algorithm used is based on a recursive approach. In this case, apart from the availability of smart meter readings, the minimum information required is the geographical position of each unit (load, micro-generation unit or storage device) as well as the phase to which it is connected.

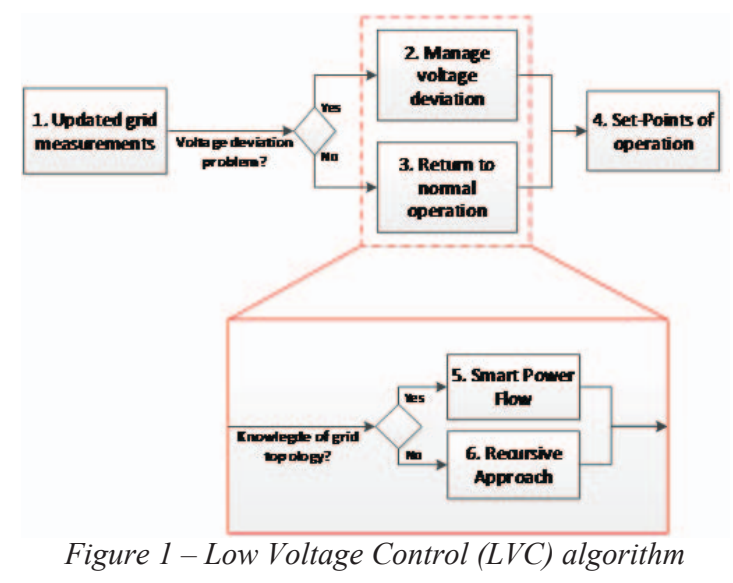

Each LV grid is managed by a DTC that is responsible for managing the different DER downstream of the corresponding $\mathrm{MV} / \mathrm{LV}$ secondary substation, as described in [1]. Each DTC may incorporate its own set of rules depending on the characteristics and degree of knowledge of the LV grid, amount and type of DER present, etc. The smart meters installed at the customer's level are used as a gateway to monitor and control these local resources, either load or generation. The proposed voltage control algorithm is assumed to be installed in each the DTC in order to manage the downstream LV grid.

\section{VOLTAGE CONTROL DEMONSTRATOR SITE}

The chosen location to test this approach was a secondary substation in a rural area located at the northeast of Évora district. The selected feeder has 12 loads ( 3 single phase and 9 three phase) and $3 \mu \mathrm{G}$ units (single phase injection, total installed capacity of $10,6 \mathrm{kVA}$ ), representing $8,1 \%$ of the feeder total hired power $(131,1 \mathrm{kVA})$ and $6,6 \%$ of the transformer capacity $(160 \mathrm{kVA})$.

Since all connected DER were privately owned and could not be controlled due to regulatory reasons, it was necessary to install additional resources to prove the SuSTAINABLE concepts. A small controllable power generating station comprising 30x250 WP (7 kW) solar panels was installed. Additionally, a small controllable 
storage station comprising $64 \times 12 \mathrm{~V} 12 \mathrm{Ah}$ electrical batteries, corresponding to a rated capacity of $9,2 \mathrm{kWh}$ ( 2 sets of $4,6 \mathrm{kWh}$ ), was installed. Figure 2 shows the PV panels and 2 of the total 8 packs of batteries installed for the showcase.

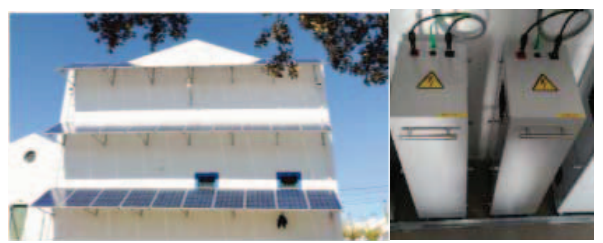

Figure 2-PV panels and 2 of the 8 battery packs installed

These resources were installed in a warehouse located at the end of the feeder (approx. 500m away from the $\mathrm{MV} / \mathrm{LV}$ substation) which represents the worst possible location in terms of its voltage impact.

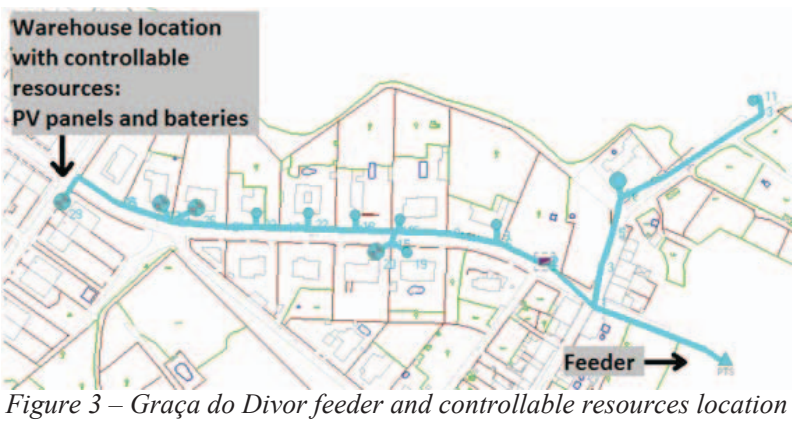

Figure 3 shows a geographical representation of the selected LV feeder, with the controllable DER.

In order to apply the proposed algorithms and test the concept 4 smart inverters developed in INESC TEC, 2 specifically for interfacing the PV generation (shown in Figure 4) and other 2 different ones for interfacing the battery storage units, were employed. The inverters have active power/voltage droop control embedded in their hardware, for local voltage control purposes, and have the capability to receive external set-points and perform according to them.

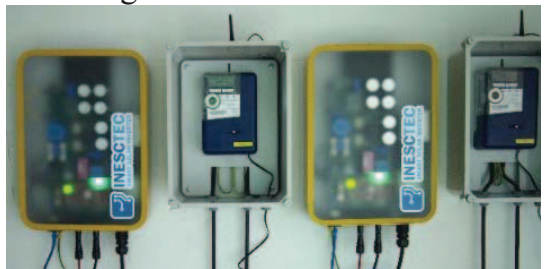

Figure 4 - Smart solar inverter prototypes and smart-meters installed

Four smart-meters were connected between the network and the inverters which, besides its normal metering function, communicate with the DTC, installed in the secondary substation, where the centralized voltage control algorithm is embedded. The DTC collects data from all the smart-meters every $5 \mathrm{~min}$. and, if necessary, sends back set-points to the controllable devices through the communication link of its respective smart-meters. Figure 5 shows an overview of the communication and interfaces applied.

In terms of communication and interfaces the smart meters are connected to the inverters via a local interface: the HAN interface while it connects to the DTC via GPRS communication. The HAN interface is present in all of EDP's smart meters and allows the smart meter to serve as a gateway to the customer's house. The communication protocol used in this interface is Modbus which is a request/reply protocol and offers several services.

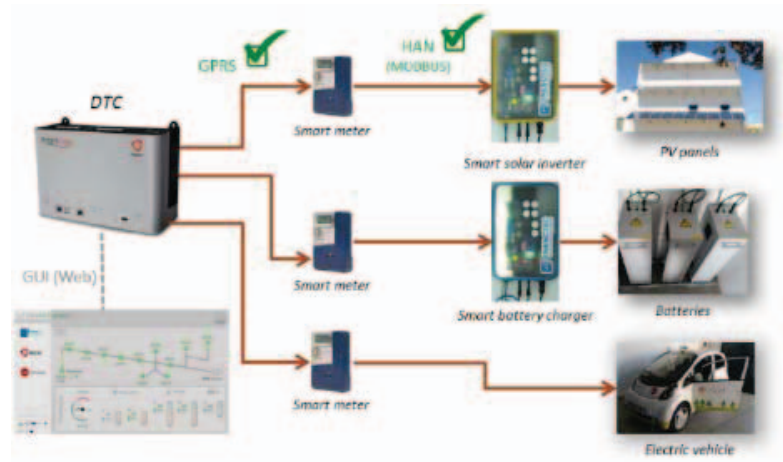

Figure 5-Communications and interfaces setup in Graça do Divor

A specific web software interface with a synoptic interface to interact with the controllable resources were developed in the DTC. The synoptic was design to display all the smart meters' data and to interact in a user friendly manner with the system.

\section{RESULTS FROM DEMONSTRATION}

Leveraging the flexibility of the assets deployed on the demo site, the LVC tool incorporated on the DTC collects data from all the meters at every 5 minutes and, in case there is any voltage violation, it initiates the algorithm in order to determine the set-point for the most suitable resource in order to mitigate that specific problem.

In Figure 6, the voltage profile (top part of the figure) and the operation profile of the controllable resources is shown for 1 full day (bottom part of the figure).

The most relevant time frame, for the purpose of assessing the performance of the algorithm, is comprehended between 09:00 and 18:00. Therefore, a detailed close-up of this period of operation is shown in Figure 7.

During this representative day, around 10:00AM, the sun conditions allowed the PV panels to start injecting power into the grid, causing a voltage rise effect, reaching the voltage limit established $(256 \mathrm{~V})$. At this point, the voltages were being controlled through the solar inverters droop control, which limited the power injection in order to keep the voltage values below the established limit. When the algorithm was enabled, the voltage limits were defined to be within the $[210 ; 246]$ $\mathrm{V}$ interval in order to prevent the voltage to rise beyond this values. 
The main events in Figure 7 are highlighted in red and numbered from 1 to 6 .

At approximately 11:45 the centralized control algorithm was enabled (Arrow 1) and detected an overvoltage. 5 minutes latter (following the running and refresh cycle of the information), the algorithm computed and sent a set-point to the EV in order to start charging (Arrow 2), as the dark green curve in Figure 7 illustrates.

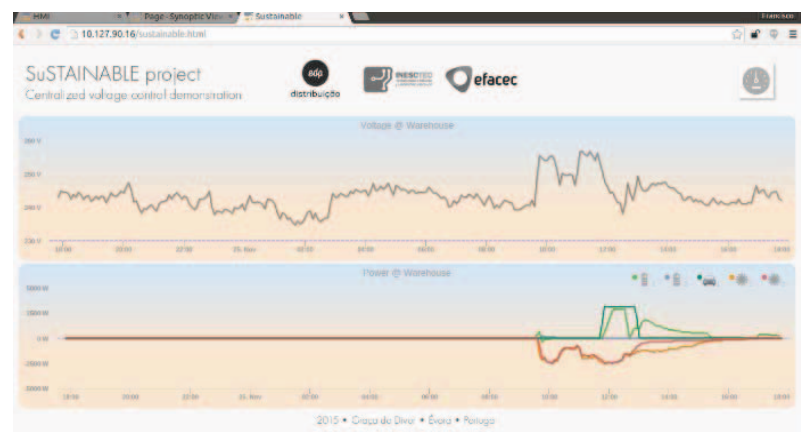

Figure 6-Detailed voltage and load profiles

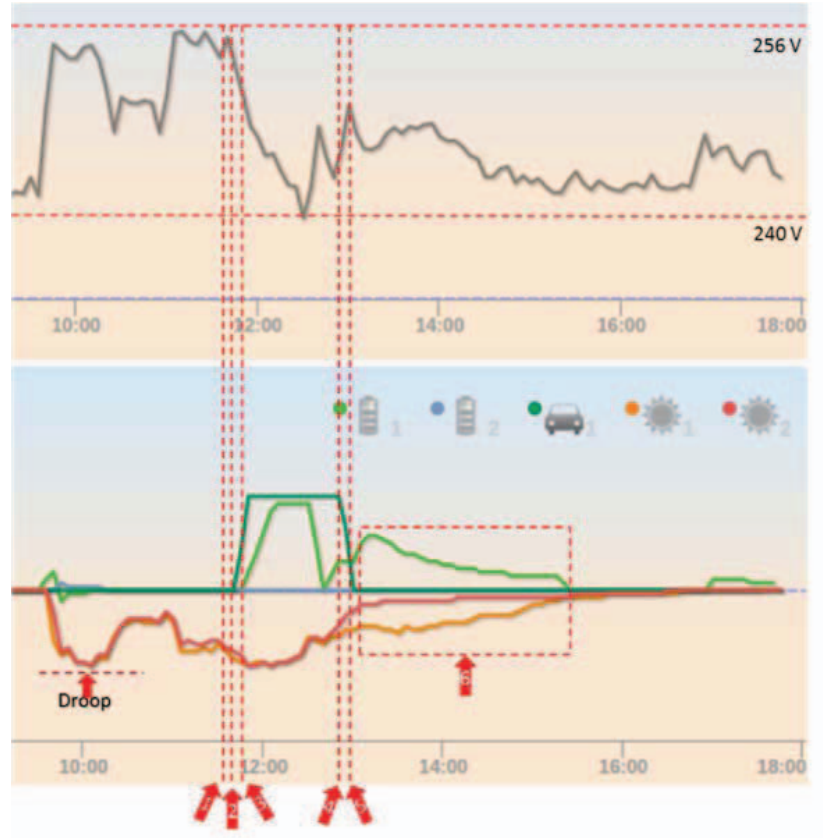

Figure 7 -Detail of operation during the demonstration

Since the voltage value was still high, 5 minutes later (the time necessary to get the synchronized measurements for all the smart meters), a new set-point was calculated. This time, the set-point was directed to the storage device 1 in order to also start charging (Arrow 3), according to the priority list of the assets to be controlled.

Then, the EV was manually disconnected (Arrow 4), as if a client had left home with the car, with the objective of observing how the algorithm would react and which solutions could be undertaken to manage the voltage deviations without that resource.
As it can be observed, after this decrease on the load (disconnection of the EV), the voltage level raised again and the algorithm computed a new set-point for the storage device 1 (Arrow 5) increasing its charging power.

As the sun started to fade and due to passing clouds, the injecting power starts to decrease and so does the voltage profile. As a result of these conditions, the previously imposed set-point could be lifted without causing voltage deviations. Arrow 6 in Figure 7 indicates a period where the charging power of storage device 1 was continuously decreasing as a result of the successive iterations of the algorithm.

An indicator for voltage variation and another for the RES cut-off, due to overvoltage, were calculated using the methodology described in [4], for KPI assessment.

\begin{tabular}{lllc}
\multicolumn{4}{c}{ Table 1 - Voltage and Power Quality performance } \\
& $\boldsymbol{d}_{\boldsymbol{v}}(\boldsymbol{S} \boldsymbol{G})$ & $\boldsymbol{d}_{\boldsymbol{v}}(\boldsymbol{B C})$ & $\Delta \boldsymbol{d}_{\boldsymbol{v}}$ \\
ENTIRE NETWORK & $4.88 \%$ & $4.94 \%$ & $\mathbf{- 1 . 0 5 \%}$ \\
CONTROLLABLE RES & $5.58 \%$ & $5.77 \%$ & $\mathbf{- 3 . 2 3 \%}$
\end{tabular}

\begin{tabular}{lccc}
\multicolumn{4}{c}{ Table 2-Reduction in RES cut-off } \\
& $\boldsymbol{E}_{\boldsymbol{S G}}$ & $\boldsymbol{E}_{\boldsymbol{B C}}$ & $\Delta \boldsymbol{E}$ \\
ENTIRE NETWORK & 2.22 & 4.22 & $\mathbf{4 7 . 4 \%}$ \\
CONTROLLABLE RES & 5.44 & 8.96 & $\mathbf{3 9 . 3 \%}$
\end{tabular}

The influence of controllable RES on the voltage variations of the network was small. However, it was proven that controllable RES have a substantially less cut-off energy. Controllable RES can maximize the energy inputted into the network.

\section{CONCLUSIONS}

The SuSTAINABLE project has proven that it is possible to have controllable DER in the LV network. If the DER is adequately controlled the amount of energy curtailed due to network restrictions is largely reduced. This has been shown by using an algorithm developed for the SUSTAINABLE project and implemented in a real LV network with controllable RES and uncontrollable RES.

\section{REFERENCES}

[1] R. Bessa, A. Madureira, L. Seca, J. Pereira, A.A. Messias, D.A. Lopes and P.G. Matos, 2015, "Advanced system architecture and algorithms for smart distribution grids: The SuSTAINABLE approach", Proceedings of the 23rd International Conference on Electricity Distribution (CIRED 2015), Lyon, France.

[2] A. Madureira, R. Bessa, J. Meirinhos, D. Fayzur, J. Filipe, A.A. Messias, D.A. Lopes and P.G. Matos, 2015, "The Impact of Solar Power Forecast Errors on Voltage Control in Smart Distribution Grids", Proceedings of the 23rd International Conference 
on Electricity Distribution (CIRED 2015), Lyon, France.

[3] J. Meirinhos, C. Gouveia, H. Costa, et al., 2014, "Description of Pre-prototype of the MultiTemporal Operational Management Tool for the MV /LV Distribution Grid”, EU Project SuSTAINABLE - Deliverable D3.4.

[4] N. Hatziargyriou, S. Papathanassiou, et al., 2013, "KPI Assessment Methodology", EU Project SuSTAINABLE - Deliverable D2.4. 